\title{
Tunable Lotus-Leaf and Rose-Petal Effects via Graphene Paper Origami
}

\author{
Changyong Cao, ${ }^{1,2 \#}$, Yaying Feng ${ }^{1 \#}$, Jianfeng Zang ${ }^{1,3,4 \#}$, Gabriel P. Lopez ${ }^{1,2,5}$, and Xuanhe \\ Zhao ${ }^{1,6,7 *}$
}

${ }^{1}$ Department of Mechanical Engineering and Materials Science, Duke University, Durham, NC 27708, USA; ${ }^{2}$ NSF Research Triangle Materials Research Science \& Engineering Center, Duke University, Durham, NC, 27708, USA; ${ }^{3}$ School of Optical and Electronic Information, Huazhong University of Science and Technology, Wuhan, Hubei 430074, China; ${ }^{4}$ Innovation Institute, Huazhong University of Science and Technology, Wuhan, Hubei 430074, China; ${ }^{5}$ Department of Biomedical Engineering, Duke University, Durham, NC, 27708, USA; ${ }^{6}$ Soft Active Materials Laboratory, Department of Mechanical Engineering, Massachusetts Institute of Technology, Cambridge, MA 02139, USA; ${ }^{7}$ Department of Civil and Environmental Engineering, Massachusetts Institute of Technology, Cambridge, MA 02139, USA.

*To whom correspondence should be addressed. Email: zhaox@ mit.edu $\left[{ }^{\#}\right]$ These authors contributed equally to this work.

Abstract: Whereas water drops on both lotus leafs and rose petals have high contact angles, the drops can easily roll off lotus leafs but strongly adhere to rose petals. Here, we report a simple and cost-effective approach to fabricate highly stretchable large-area surfaces that give lotus-leaf and rose-petal effects by harnessing origami patterns formed in graphene paper (GP) bonded on a pre-strained elastomer substrate. The surfaces of the GP origami exhibit high contact angles $\left(>160^{\circ}\right)$ yet robust adhesion to water drops. After depositing a gold film of a few nanometers on the GP, the origami of GP-Au gives high contact angle $\left(>160^{\circ}\right)$ and low roll-off angles $\left(<5^{\circ}\right)$ of water drops. We show that the high surface roughness of the GP origami leads to the high contact angle of water drops, and hydrophilic groups and defects on on the GP surface significantly enhance the adhesive forces of drops on the GP origami, leading to the rose-petal effect. The coating of gold film does not affect the surface roughness of the origami but significantly reduces the adhesive force, transitioning the rose-petal to 
lotus-leaf effects. In addition, the wetting properties of both GP and GP-Au origamis can be tuned over a wide range by simply stretching or compressing the substrate elastomer.

Keywords: tunable adhesion, superhydrophobicity, graphene paper, origami, rose-petal effect, lotus-leaf effect

\section{Introduction}

Whereas water drops on both lotus leafs and rose petals have high contact angles, the drops can easily roll off lotus leafs but strongly adhere to rose petals (Fig. 1a and b) $[1,2]$. The wetting properties of lotus leafs and rose petals, attributed to a combination of nano- to microscale topographies and chemical compositions of their surfaces, have been intensively studied [3-5]. In particular, the so-called 'lotus-leaf effect' (Fig. 1a) and 'rose-petal effect' (Fig. 1b) have motivated researchers to create novel surfaces with high contact angles and low or high adhesions to water drops, for applications as diverse as self-cleaning and antifogging surfaces, fluid drag reduction, humidity control for electronic devices, dry adhesives, and no-loss transport of liquid microdroplets [2, 3, 6-8]. Existing fabrication methods for surfaces with lotus-leaf or rose-petal effects include template-wetting method, lithography methods, ultrafast (femtosecond) laser irradiation, anisotropic etching, plasma treatment and chemical vapor deposition (CVD) based surface method [4, 5, 9-13]. These methods have their own limitations to some extent. For instance, the broadly used lithography methods are usually confined in a relatively small sample area and involve multiple fabrication steps that incur high cost [5]. Although the template-wetting method may be potentially used for large-area applications, it is difficult to make surfaces with tunable wetting capability. In addition, most of these methods are not applicable to surfaces that require high stretchability and deformability [11]. 
In this work, we report a simple approach to fabricate highly stretchable large-area surfaces that give either lotus-leaf effect or rose-petal effect, by harnessing origami patterns in graphene paper (GP) bonded on a pre-strained elastomer substrate. The GP used for generating the origami patterns is prepared by chemical reduction of graphene oxide (GO), a low-cost and effective method for producing large-area graphene papers [14]. We demonstrate that the surfaces of GP origami exhibit high contact angles $\left(>160^{\circ}\right)$ and robust adhesion to water drops, giving the rose petal effect. Interestingly, by depositing a gold film of a few nanometers on the GP, the origami of GP-Au keeps the same topologies as those of GP origami, but giving high contact angles $\left(>160^{\circ}\right)$ and low roll-off angles $\left(<5^{\circ}\right)$, i.e., the lotus-leaf effect. Moreover, the wetting properties of both GP and GP-Au origamis can be tuned over a wide range by simply stretching or compressing the elastomer substrate. We further discuss the effects of chemical properties and surface morphologies of the GP and GP$\mathrm{Au}$ origamis on their wetting properties. Our method provides a simple strategy to develop novel stretchable large-area surfaces with tunable adhesions to water drops.

\section{Fabrication procedure of graphene-paper origami}

Origami patterns can be generated by biaxial compression of a thin elastic film bonded on a compliant substrate [15-23]. Fig. S1 illustrates the procedure for fabricating GP origami by harnessing mechanical instabilities of GP compressed on a pre-strained elastomer substrate. The GP was made by vacuum filtration of the graphene oxide (GO) solution through an nylon membrane filter followed by air drying and peeling from the filter [24] (see Fig. S1 and Materials and Methods in Supplementary Materials). The thickness of GO paper can be tuned from a few nanometers to several micrometers by varying the mass of GO in solution during vacuum filtration. Meanwhile, a square-shaped elastomer film, VHB acrylic 4910 with thickness of $1 \mathrm{~mm}$ (3M Inc., US), was biaxially stretched along two orthogonal in-plane 
directions by engineering strains of $\varepsilon_{\text {pre } 1}$ and $\varepsilon_{\text {pre } 2}$. Thanks to the high stretchability of the elastomer, the pre-strains can be set to values ranging from $100 \%$ to $400 \%$ in our experiments. The paper-like GO film (50-500 $\mathrm{nm}$ thick) was then transferred to pre-strained elastomer substrate by a dry transfer method due to the lower adhesion energy between GO film and filter membrane than that between GO film and target substrate. The as-prepared GO paper (Fig. 1c) was then reduced in saturated vapor of Hydrazine monohydrate (N2H4 64-65\%, reagent grade, $98 \%$, Sigma- ALDRICH) at $80^{\circ} \mathrm{C}$ for $4 \sim 6$ hours [25]. Thereafter, the pre-strains in the elastomer substrate were relaxed uniaxially or biaxially to generate parallel ridges (Fig. 1d) or hierarchical origami patterns (Fig. 1f-h) [10, 17].

\section{Results and discussion}

\subsection{Patterns of GP origami}

To discuss the origami patterns generated by uniaxial or biaxial compression of film-substrate system, we define the strains in the GP and the substrate as follow. Denote the lateral dimensions of the GP along two pre-strained directions as $L_{1}$ and $L_{2}$ at the initial transferred state and as $l_{1}$ and $l_{2}$ at the relaxed state. The nominal compressive strains in the GP are defined as $\varepsilon_{f 1}=\left(L_{1}-l_{1}\right) / L_{1}$ and $\varepsilon_{f 2}=\left(L_{2}-l_{2}\right) / L_{2}$. We further denote the tensile strains in the substrate at a state as $\varepsilon_{s 1}$ and $\varepsilon_{s 2}$. Thus, the nominal compressive strains in the GP film and the tensile strains in the substrate are related by

$$
\begin{aligned}
& \varepsilon_{f 1}=\left(\varepsilon_{\text {prel }}-\varepsilon_{s 1}\right) /\left(\varepsilon_{\text {pre } 1}+1\right) \\
& \varepsilon_{f 2}=\left(\varepsilon_{\text {pre } 2}-\varepsilon_{s 2}\right) /\left(\varepsilon_{\text {pre } 2}+1\right)
\end{aligned}
$$

When the substrate is fully relaxed (i.e., $\varepsilon_{s 1}=\varepsilon_{s 2}=0$ ), the nominal compressive strains in the

GP reach the maximum values of $\varepsilon_{\text {prel }} /\left(\varepsilon_{\text {prel }}+1\right)$ and $\varepsilon_{\text {pre } 2} /\left(\varepsilon_{\text {pre } 2}+1\right)$, respectively [10]. 
We next discuss the surface patterns of GP generated by releasing the pre-strained elastomer substrates (i.e., $\varepsilon_{\text {prel }}=\varepsilon_{\text {pre } 2}=300 \%$ ). The biaxial pre-strains in the substrate are relaxed either sequentially or simultaneously along two orthogonal pre-strained directions [10, 16]. During sequential relaxation of the substrate, the pre-strain $\varepsilon_{\text {prel }}$ is first released, so that a pattern of ridges develops in the GP on elastomer substrate. As reported in a previous work, the wavelength and amplitudes of ridges on fully relaxed substrate follow the scaling law $\lambda_{\text {ridge }} / H_{f} \propto\left(\mu_{f} / \mu_{s}\right)^{0.31}$ and $A_{\text {ridge }} / H_{f} \propto\left(\mu_{f} / \mu_{s}\right)^{0.31}$, respectively; and the aspect ratio (i.e., height over wavelength) of the ridge follows $A_{\text {ridge }} / \lambda_{\text {ridge }} \approx 0.52 \varepsilon_{\text {pre }}+0.23$, which was obtained from numerical simulations and validated by experimental data [10]. Since $\varepsilon_{\text {prel }}=300 \%$ in the current study, the predicted aspect ratio of the ridge should be $\sim 1.79$. As shown on Fig. 1e, the aspect ratio of the ridges is indeed $\sim 1.64$, much higher than typical aspect ratios of wrinkles, creases and folds. In addition, due to the large deformation in the elastomer substrate under the ridges, partial delamination may occur at the top region of the ridges, as shown in Fig. 1e) [10, 26].

When the pre-strain $\varepsilon_{\text {pre } 2}$ is subsequently released, the pattern of parallel ridges will be deformed as an effective layer with thickness larger than the GP film, leading to a pattern of second-level ridges orthogonal to the previous first-level ridges (Figs. 1f and $\mathbf{1 h}$ ). On the other hand, simultaneously relaxing the substrate generates randomly distributed hierarchical structures significantly different from those of sequentially-relaxed ones. The intersection of two ridges generates a vertex with a sharp tip. As the biaxial pre-strains are fully relaxed, the surface is featured with a pattern of buckled ridges together with sharp vertices almost evenly distributed on the surface (Fig. 1g).

To better understand the evolution of the origami patterns, we develop a finite-element model for the GP-elastomer substrate system (Fig. 2). The mechanical properties of GP film 
and VHB elastomer substrate are measured by a Micro-Strain Analyzer under uniaxial tensions. The stress vs. stretch data are then fitted to the neo-Hookean model to obtain shear moduli of $\mu_{f}=19 \mathrm{MPa}$ and $\mu_{s}=20 \mathrm{kPa}$ for GP and the VHB elastomer, respectively (Fig. S2a and S2b). The adhesion energy between GP and VHB are measured to be $580 \mathrm{~J} / \mathrm{m}^{2}$ (Figs. S2c, S2d and S3). The elastomer substrate (1 mm thick) is treated as infinitely thick in the finite-element model, since it is much thicker than the GP $(\sim 0.1 \mu \mathrm{m}$ thick $)$. Fig. 2a shows the initiation and evolution of localized ridges in a GP-elastomer substrate structure with prestrain in the subtrate $\varepsilon_{\text {prel }}=300 \%$. As the uniaxial pre-strain in the substrate is relaxed, a compressive strain $\varepsilon_{f 1}$ in the GP film will be generated, and when the compressive strain in the GP film exceeds a critical value, a series of sinusoidal wrinkles set in. As compressive strain $\varepsilon_{f 1}$ further increases, the amplitude of certain wrinkles increase much more dramatically than others, leading to a combination of wrinkles and localized ridges. With further increment of $\varepsilon_{f 1}$, more ridges form on the surface and the amplitude of the ridges increases (Fig. 2a). The compressive strains $\boldsymbol{\varepsilon}_{f 1}$ in the GP varies from $0 \%$ to $75 \%$ when the pre-strain in the substrate is relaxed from $300 \%$ to $0 \%$ (Fig. 2a).

Since the deformation in the elastomer substrate is excessively high around peaks of the ridges, delamination between GP and the substrate has been observed around these locations (Fig. 2b and 2c). To calculate the length of delamination, we assume that the substrate has delaminated from the GP film at the each peak of the ridge symmetrically in the finite-element model, as shown in Fig. 2a. The potential energy of each ridge (i.e., over one wavelength) with the delamination at equilibrium state can be calculated as $\Pi=U_{f}+U_{s}+\Gamma D[27,28]$, where $U_{f}$ and $U_{s}$ are the elastic energies of the film and the substrate, $\Gamma$ the film-substrate adhesion energy of the film, and $D$ the delaminated length measured on the film. The formation of delamination will decrease the elastic energy of the 
structure by relaxing the substrate, but increase the adhesion energy of the system. Therefore, at a certain delaminated length $D_{c}$, the potential energy of the system can reach a minimum. By adopting the Maxwell stability criterion, we regard $D_{c}$ as the stable delaminated length at each peak of the ridge. Fig. 2d shows the normalized potential energy $\Pi / \Pi_{0}$ as a function of the normalized delamination size $D /\left[\lambda\left(1+\varepsilon_{\text {pre } 1}\right)\right]$ for different adhesion energies between substrate and film, where $\Pi_{0}$ denotes the potential energy of the ridged film-substrate structure without delamination. The minimum point shown on each curve indicates the predicted delamination size for the given adhesion energy. It can be seen that the normalized delamination size decreases with the increase of the adhesion strength of the interface (Fig. 2e). When $\Gamma=580 \mathrm{~J} / \mathrm{m}^{2}$, our model predicts that $D_{c} /\left\lfloor\lambda\left(1+\varepsilon_{\text {prel }}\right)\right\rfloor \approx 0.28$, which is consistent with the experimental observation $\mathrm{D} /\left[\lambda\left(1+\varepsilon_{\text {pre } 1}\right)\right] \approx 0.30$, where the $\mathrm{D}$ is the length of the GP debonded from the VHB substrate in the experimental image, and is measured using the ImageJ software package. (Fig. 2b).

\subsection{Rose-petal effect of the GP origami}

To generate the GP origami, we prepare samples by sequentially relaxing elastomer substrates with $\varepsilon_{\text {prel }}=\varepsilon_{\text {pre } 2}=300 \%$. The thickness of the GPs is $\sim 0.1 \mu \mathrm{m}$ unless otherwise specified. Prior to relaxing the substrate, the obtained GP is treated with $1 \mathrm{H}, 1 \mathrm{H}, 2 \mathrm{H}, 2 \mathrm{H}-$ Perfluorodecyltriethoxysilane (PFTES, 97\%, ALDERCH Chemistry, USA) by gas-phase evaporation in a desiccator under vacuum for 4 hours to couple hydrophobic functional groups on the GP surfaces [29]. The wettability of the GP origami surface is characterized by the static contact angles, roll-off angles, advancing and receding angles. Contact angle hysteresis, i.e., the difference between advancing and receding angles, is also calculated to indicate the adhesion of water drop on the GP origami surfaces $[11,30]$. 
From Fig. 3a, it can be seen that the static contact angle of a sessile water drop reaches over $160^{\circ}$ on the GP origami, while the contact angle hysteresis can reach over $155^{\circ}$ (Figs. 3c and 3d), indicating the rose-petal effect [31]. Fig. 3b shows that a water drop of $5 \mu \mathrm{L}$ maintains high contact angle of $160^{\circ}$ on the GP origami but still being pinned on the surface even when the sample is turned upside down. The strong adhesion of water drop on the GP origami surface is also demonstrated in Fig. 3e, in which a water drop with much higher volume (40 $\mu \mathrm{L}$ ), with a gravity of $392 \mu \mathrm{N}$, can be firmly suspended on the vertically placed GP-origami surface.

The strong adhesion of water drop to the GP origami may result from residual hydrophilic groups and structural defects on the GP [32]. Since the wettability of a surface is significantly affected by the outermost chemical groups of the surface, certain residual hydrophilic groups (e.g., - $\mathrm{OH}$ or $-\mathrm{COOH}$ ) introduced in chemical processing [33] may remain on the surface of GP and makes the water partially penetrate into the valleys of the surface, significantly increasing its adhesion to water drops. In addition, defects such as pores and facial edges (Fig. 1c, 1f and Fig. S4) may be introduced in the GP fabricated with the filtration method $[34,35]$. These defects lead to high local surface energy and may penetrate into the sessile water drop so as to increase the adhesion force [32].

In addition, we find that both the contact angle and contact angle hysteresis of drops on the GP origami surface can be tuned over a very wide range by simply stretching or compressing the substrate. From Fig. 3f, it can be seen that the static contact angle of water drop on GP surface reduces from $163^{\circ}$ to $93^{\circ}$ and the contact angle hysteresis decrease from $157^{\circ}$ to $85^{\circ}$, when the biaxial tensile strain in the substrate increases from $0 \%$ to $300 \%$ (or the biaxial compressive strain in the GP decreases from $75 \%$ to $0 \%$ ). These variations are mainly due to the change of the surface roughness of GP under deformation. The decrease of compressive strain in the GP by stretching the elastomer substrate can drastically reduce the surface rough of the GP and therefore lead to the decrease of static contact angle (Fig. 3f). 
The static contact angle of surfaces with rose-petal effect can be characterized by the Cassie-Baxter equation [2, 22, 36-38]

$$
\cos \theta=r_{f} f \cos \theta_{0}+f-1
$$

where $\theta$ is the apparent contact angle of the origami surface, $\theta_{0}$ intrinsic contact angle of flat GP surface, $f$ is the fraction of the projected area of the solid surface that is wetted by the liquid, and $r_{f}$ is the roughness ratio of the wet area. The roughness ratio of the GP origami can be calculated as $r_{f}=L_{1} L_{2} /\left(l_{1} l_{2}\right)=1 /\left[\left(1-\varepsilon_{f 1}\right)\left(1-\varepsilon_{f 2}\right)\right][16]$. Since the pre-strain in the substrate is $\varepsilon_{\text {prel }}=\varepsilon_{\text {pre } 2}=300 \%$, we can calculate $\varepsilon_{f 1}=\varepsilon_{f 2}=0.75$ and $r_{f}=16$ at the fully relaxed state of the substrate. Since the measured $\theta=163^{\circ}$ at the fully relaxed state of the substrate and $\theta_{0}=93^{\circ}$, we further calculate $f=0.27$, indicating that a small portion of the solid surface is wetted. As the compressive strain in GP is released (i.e., the origami surface is unfolded), the contact angle of GP origami reduces to approximately the intrinsic contact angle $\theta_{0}=93^{\circ}$. On the other hand, the receding angle of water drop on the GP is very low $\left(<16^{\circ}\right)$ regardless of the compressive strains on the GP, owning to the strong adhesion of water drop on the GP (Fig. 3g). Since the advancing angle of water drop on GP is approximately equal to its static contact angle, the contact angle hysteresis also reduces with the decrease of compressive strain and surface roughness of the GP (Fig. 3g).

\subsection{Lotus-leaf effect of the GP origami coated with gold nanofilm.}

To achieve the lotus-leaf effect, we deposit a layer of gold film with thickness of $3 \pm 1 \mathrm{~nm}$ on the GP (Fig. 4a). Since the gold nanofilm is much thinner than the GP, it does not significantly affect the structural features of the GP origami (Fig. 1f, 1h, and Fig. S5). The gold-coated GP is further immersed into (heptadecafl uoro-1,1,2,2-tetrahydrodecyl) trichlorosilane vapor for 4 hours to be hydrophobilized. Since the gold nanofilm can cover 
chemical groups and nanoscale defects on the GP, the GP coated with gold nanofilm can be more homogeneously hydrophobilized than bare GP samples. Therefore, it is expected that the adhesion of water drops on the GP-Au origami is lower than that on the GP origami.

The wetting property of the gold-coated GP origami is characterized following procedures described in the previous section. The static contact angle of a water drop on the GP origami coated with gold nanofilm can reach $168^{\circ}$ (Fig. 4b), and the water drops can easily roll off at a small tilting angle of $5^{\circ}$ (Fig. 4c), giving the lotus-leaf effect. The high contact angles of GP-Au origami pattern can be characterized by the Cassie-Baxter model of Eq.(2), in which the roughness of the surface on fully relaxed substrate $r_{f}=12.25$ (for $\varepsilon_{\text {prel }}=\varepsilon_{\text {pre } 2}=250 \%$ ) and the fraction of the projected area of the solid surface that is wetted by the liquid $f=0.15$, lower than the fraction of GP origami without Au coating. Furthermore, when the biaxial tensile strain in the substrate is confined within $100 \%$, the static contact angle of water drop can stay above $150^{\circ}$ and roll-off angle below $10^{\circ}$ (Fig. $4 \mathbf{d}$ ). Consistently, the contact angle hysteresis maintains below $15^{\circ}$ when the tensile strain in substrate increases from $0 \%$ to $120 \%$ (Fig. 4e). If the substrate is biaxially stretched beyond $100 \%$ strain, the contact angle of water drops would quickly decrease to $124^{\circ}$ while the rolloff angle increases to $90^{\circ}$ due to significant diminishment of surface structure (Fig. $\left.4 \mathbf{d}\right)$.

\subsection{Reversibility and repeatability of the tunable lotus-leaf and rose-petal effects}

We further demonstrate that the tunable lotus-leaf and rose-petal effects of the GP origami are reversible and repeatable under cyclic deformation of the elastomer substrate. We repeatedly stretch and relax the elastomer substrate with GP origami to prescribed strains, and measure the contact angle and contact angle hysteresis of water drops on the GP origami. As shown in Fig. 5, the variations of contact angle and contact angle hysteresis for both GP origami and gold-coated GP origami are reversible and repeatable over multiple stretch-relax cycles. 
These rose-petal or lotus-leaf coatings capable of reversible and repeatable tunability can potentially find a variety of applications, such as manipulation and transport of liquid microdrops, biological and chemical analysis and detection, and microfluidic lab-on-a chip devices $[12,39,40]$.

\section{Conclusion}

In summary, we have demonstrated a new cost-effective approach to fabricate highly stretchable, large-area surfaces that give either rose-petal effect or lotus-leaf effect by harnessing the GP origami formed on a prestretched elastomer substrate. The surface of GP origami exhibits a high static contact angle $\left(>160^{\circ}\right)$, high contact angle hysteresis $\left(>150^{\circ}\right)$, and high adhesive force to water drops, giving the rose-petal effect. On the other hand, after coated with a gold nanofilm, GP origami transits to be a super-hydrophobic surface with lotus-leaf effect. The functional groups affixed on the GP surface, capillary adhesion and structural defects on the GP surface contribute to the robust adhesion of the GP origami. Nevertheless, the coating of gold nanofilm covers the chemical groups, defects and edges on the GP origami surfaces, and greatly reduces the adhesion of GP origami to water drops, leading to the transition from rose-petal to lotus-leaf effect. We finally demonstrate that the tunable wetting properties and adhesion of GP origami surfaces are reversible and repeatable under multiple cycles of deformation.

\section{Acknowledgements}

The work was financially supported by ONR (N00014-14-1-0619), NSF (CMMI-1253495, DMR-1121107). J.Z. thanks the support from the National 1000 Talents Program of China tenable in Huazhong University of Science and Technology (HUST), China. Y.F. thanks Prof. Stefan Zauscher at Duke University for his helpful discussion and support. G.P.L 
acknowledges the financial support from ONR (N0014-13-1-0828) and NSF MRSEC (DMR1121107).

\section{Supporting Information}

Supplementary material related to this article can be found online at ScienceDirect.

\section{Reference}

1. Barthlott, W. and C. Neinhuis, Purity of the sacred lotus, or escape from contamination in biological surfaces. Planta, 1997. 202.

2. $\quad$ Feng, L., et al., Petal Effect: A Superhydrophobic State with High Adhesive Force. Langmuir, 2008. 24(8): p. 4114-4119.

3. Zhang, X., et al., Superhydrophobic surfaces: from structural control to functional application. Journal of Materials Chemistry, 2008. 18(6): p. 621-633.

4. Dawood, M., et al., Mimicking both petal and lotus effects on a single silicon substrate by tuning the wettability of nanostructured surfaces. Langmuir, 2011. 27(7): p. 41264133.

5. Yan, Y., N. Gao, and W. Barthlott, Mimicking natural superhydrophobic surfaces and grasping the wetting process: A review on recent progress in preparing superhydrophobic surfaces. Advances in colloid and interface science, 2011. 169(2): p 80-105.

6. Howarter, J.A. and J.P. Youngblood, Self-Cleaning and Anti-Fog Surfaces via Stimuli - Responsive Polymer Brushes. Advanced materials, 2007. 19(22): p. 38383843.

7. Lee, H., B.P. Lee, and P.B. Messersmith, A reversible wet/dry adhesive inspired by mussels and geckos. Nature, 2007. 448(7151): p. 338-341.

8. Orner, B.P., et al., Arrays for the Combinatorial Exploration of Cell Adhesion. Journal of the American Chemical Society, 2004. 126(35): p. 10808-10809.

9. Dorrer, C. and J. Rühe, Wetting of Silicon Nanograss: From Superhydrophilic to Superhydrophobic Surfaces. Advanced Materials, 2008. 20(1): p. 159-163.

10. Cao, C., et al., Harnessing Localized Ridges for High-Aspect-Ratio Hierarchical Patterns with Dynamic Tunability and Multifunctionality. Advanced Materials, 2014. 26(11): p. 1763-1770.

11. Balu, B., et al., Tunability of the adhesion of water drops on a superhydrophobic paper surface via selective plasma etching. Journal of Adhesion Science and Technology, 2009. 23(2): p. 361-380.

12. Lai, Y., et al., Designing superhydrophobic porous nanostructures with tunable water adhesion. Advanced Materials, 2009. 21(37): p. 3799-3803.

13. Rahmawan, Y., L. $\mathrm{Xu}$, and $\mathrm{S}$. Yang, Self-assembly of nanostructures towards transparent, superhydrophobic surfaces. Journal of Materials Chemistry A, 2013. 1(9): p. 2955-2969.

14. Ding, Y.-H., et al., Surface adhesion properties of graphene and graphene oxide studied by colloid-probe atomic force microscopy. Applied Surface Science, 2011. 258(3): p. 1077-1081.

15. Mahadevan, L. and S. Rica, Self-Organized Origami. Science, 2005. 307(5716): p. 1740. 
16. Zang, J., et al., Multifunctionality and control of the crumpling and unfolding of largearea graphene. Nat Mater, 2013. 12(4): p. 321-325.

17. Zang, J., et al., Stretchable and High-Performance Supercapacitors with Crumpled Graphene Papers. Scientific Reports, 2014. 4: p. 6492.

18. Kim, P., et al., Tunable Optical Diffuser Based on Deformable Wrinkles Rational Design of Mechano-Responsive Optical Materials by Fine Tuning the Evolution of Strain-Dependent Wrinkling Patterns. Adv. Opt. Mater., 2013. 1: p. 381-388.

19. Liu, Y., et al., Three-dimensional folding of pre-strained polymer sheets via absorption of laser light. Journal of Applied Physics, 2014. 115(20): p. 204911.

20. Efimenko, K., et al., Nested self-similar wrinkling patterns in skins. Nat Mater, 2005. 4(4): p. 293-297.

21. Lin, P.-C. and S. Yang, Spontaneous formation of one-dimensional ripples in transit to highly ordered two-dimensional herringbone structures through sequential and unequal biaxial mechanical stretching. Applied Physics Letters, 2007. 90(24): p. 241903.

22. Lin, P.-C. and S. Yang, Mechanically switchable wetting on wrinkled elastomers with dual-scale roughness. Soft Matter, 2009. 5(5): p. 1011-1018.

23. Li, B., et al., Mechanics of morphological instabilities and surface wrinkling in soft materials: a review. Soft Matter, 2012. 8(21): p. 5728-5745.

24. Dikin, D.A., et al., Preparation and characterization of graphene oxide paper. Nature, 2007. 448(7152): p. 457-460.

25. Park, S., et al., Hydrazine-reduction of graphite- and graphene oxide. Carbon, 2011. 49(9): p. 3019-3023.

26. Sun, J.-Y., et al., Folding wrinkles of a thin stiff layer on a soft substrate. Proceedings of the Royal Society A: Mathematical, Physical and Engineering Science, 2012. 468(2140): p. 932-953.

27. Chen, D., et al., Surface energy as a barrier to creasing of elastomer films: An elastic analogy to classical nucleation. Physical review letters, 2012. 109(3): p. 038001.

28. Wang, Q. and X. Zhao, A three-dimensional phase diagram of growth-induced surface instabilities. Sci. Rep., 2015. 5(8887).

29. Shu, Y., K. Krishnacharya, and L. Pei-Chun, Harnessing Surface Wrinkle Patterns in Soft Matter. Advanced Functional Materials, 2010. 20(16): p. 2550-2564.

30. Callies, M. and D. Quéré, On water repellency. Soft matter, 2005. 1(1): p. 55-61.

31. Whyman, G., E. Bormashenko, and T. Stein, The rigorous derivation of Young, Cassie-Baxter and Wenzel equations and the analysis of the contact angle hysteresis phenomenon. Chemical Physics Letters, 2008. 450(4-6): p. 355-359.

32. Tian, S., et al., Robust adhesion of flower-like few-layer graphene nanoclusters. Scientific reports, 2012. 2.

33. Chen, Z., et al., Superhydrophobic Graphene - Based Materials: Surface Construction and Functional Applications. Advanced Materials, 2013. 25(37): p. 5352-5359.

34. Feng, H., et al., A low-temperature method to produce highly reduced graphene oxide. Nat Commun, 2013. 4: p. 1539.

35. Sun, X.-Y., et al., Anisotropic vacancy-defect-induced fracture strength loss of graphene. RSC Advances, 2015. 5(18): p. 13623-13627.

36. Cassie, A. and S. Baxter, Wettability of porous surfaces. Transactions of the Faraday Society, 1944. 40: p. 546-551.

37. Checco, A., et al., Collapse and reversibility of the superhydrophobic state on nanotextured surfaces. Physical Review Letters, 2014. 112(21): p. 216101.

38. Checco, A., A. Rahman, and C.T. Black, Robust Superhydrophobicity in Large - Area Nanostructured Surfaces Defined by Block-Copolymer Self Assembly. Advanced Materials, 2014. 26(6): p. 886-891. 
39. Shreshta, J., et al., Adaptive wettability-enhanced surfaces ordered on molded etched substrates using shrink film. Smart Materials and Structures, 2013. 22(1): p. 014014.

40. Lai, Y., et al., In Situ Surface-Modification-Induced Superhydrophobic Patterns with Reversible Wettability and Adhesion. Advanced Materials, 2013. 25(12): p. 1682-1686.

41. Cao, Y. and J.W. Hutchinson, Wrinkling phenomena in neo-Hookean film/substrate bilayers. Journal of Applied Mechanics, 2012. 79(3): p. 031019 


\section{Figures and Figure Captions}
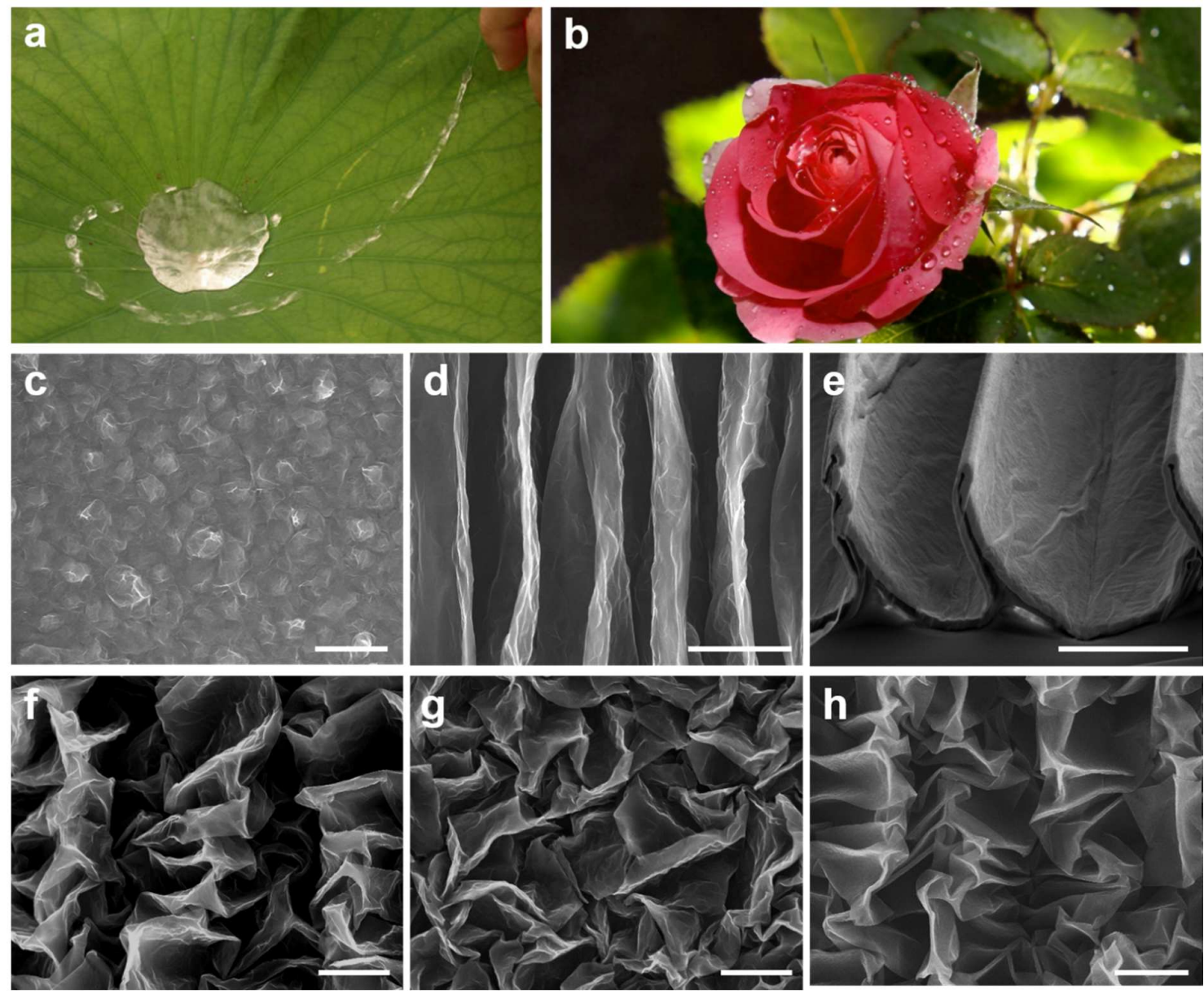

Fig. 1. Lotus-leaf and rose-petal effects achieved via GP origami. (a) Optical image of water drops rolling on a lotus leaf (Photograph courtesy of Julia Vasic). (b) Optical image of rose petals attached with water drops (Photograph courtesy of wallpaper.com). (c) SEM image of GP at flat state. (d) SEM image of localized ridges of GP formed by relaxing uniaxially pre-stretched elastomer substrate $\left(\varepsilon_{\text {prel }}=300 \%, \varepsilon_{\text {pre } 2}=0 \%\right)$. (e) SEM image of the cross-section of ridged GP by sectioning the sample with Focused Ion Beam (FIB) milling. SEM images of surface patterns of GP formed by (f) sequential relaxation or (g) simultaneous relaxation of biaxially pre-stretched elastomer substrate $\left(\varepsilon_{\text {prel }}=\varepsilon_{\text {pre } 2}=30 \%\right.$ ). (h) SEM image of surface patterns formed in GP coated with nano-gold film $(3 \pm 1 \mathrm{~nm})$ by sequential relaxation of biaxially pre-stretched elastomer substrate $\left(\varepsilon_{\text {prel }}=\varepsilon_{\text {pre } 2}=30 \%\right)$. The scale bars in (c)-(h) indicate $5 \mu \mathrm{m}$. 
a
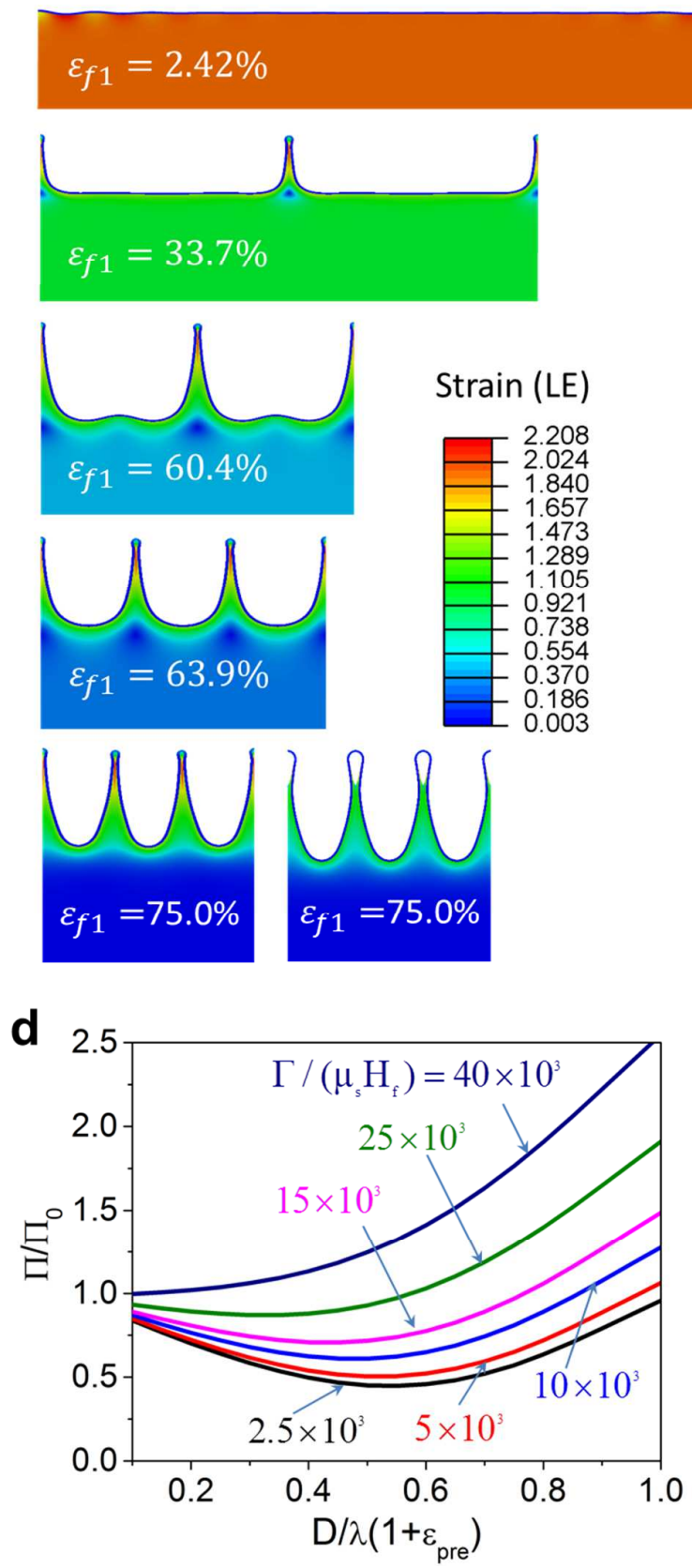
Strain (LE)

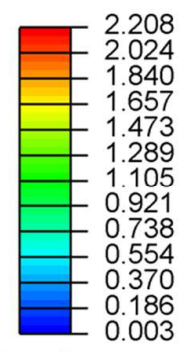

b

C

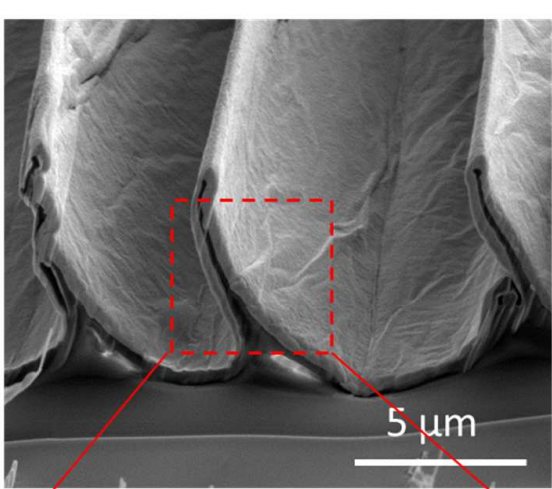

c
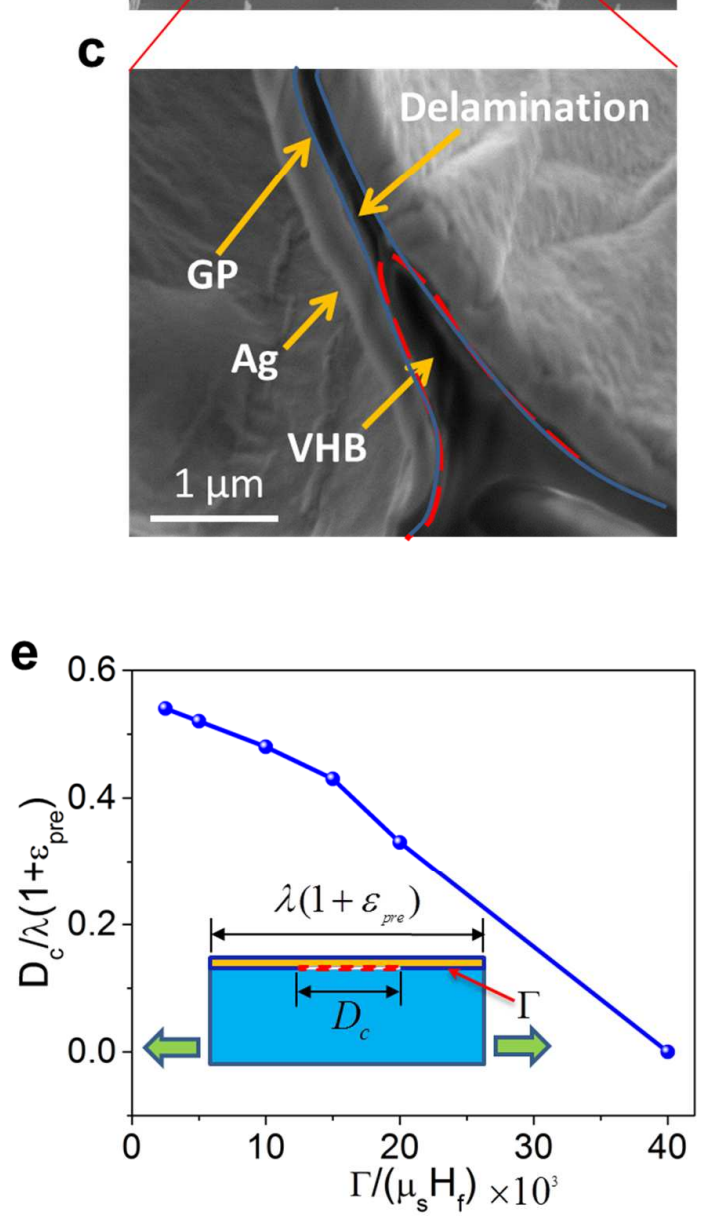

Fig. 2. Theoretical and numerical results on the evolution of localized-ridge and delamination in GP-elastomer-substrate system. (a) Distribution of maximum in-plane principal strain in the elastomer substrate during the formation of localized ridges and delamination. The thickness of GP is taken to be $0.1 \mu \mathrm{m}$, and the elastomer substrate is modeled as infinitely deep. The prestrain in the elastomer substrate is set to be $\varepsilon_{\text {pre }}=300 \%$. (b-c) SEM images of a sectioned ridge pattern of GP film on a fully relaxed substrate with an 
uniaxial pre-strain of $300 \%$. The ridged sample is sectioned by Focused Ion Beam (FIB) milling. Before sectioning the sample, we deposit $500 \mathrm{~nm}$ silver $(\mathrm{Ag})$ on top of the ridged film to prevent potential damage of the film from gallium ion irradiation. The cross-sectional profiles are imaged by the high-resolution SEM built in the FIB-system. With extremely large prestrain and lower bond strength, partial delamination may occur at the interface between GP film and elastomer substrate. (d) The normalized potential energy stored in the system as a function of normalized delamination size $D /\left[\lambda\left(1+\varepsilon_{\text {prel }}\right)\right]$. The stable delamination size for a given adhesion energy of the interface can be determined by minimizing the potential energy of the system, i.e. the minimum point on each curve. (e) The normalized delamination size as a function of adhesion energy between GP and elastomer substrate. 

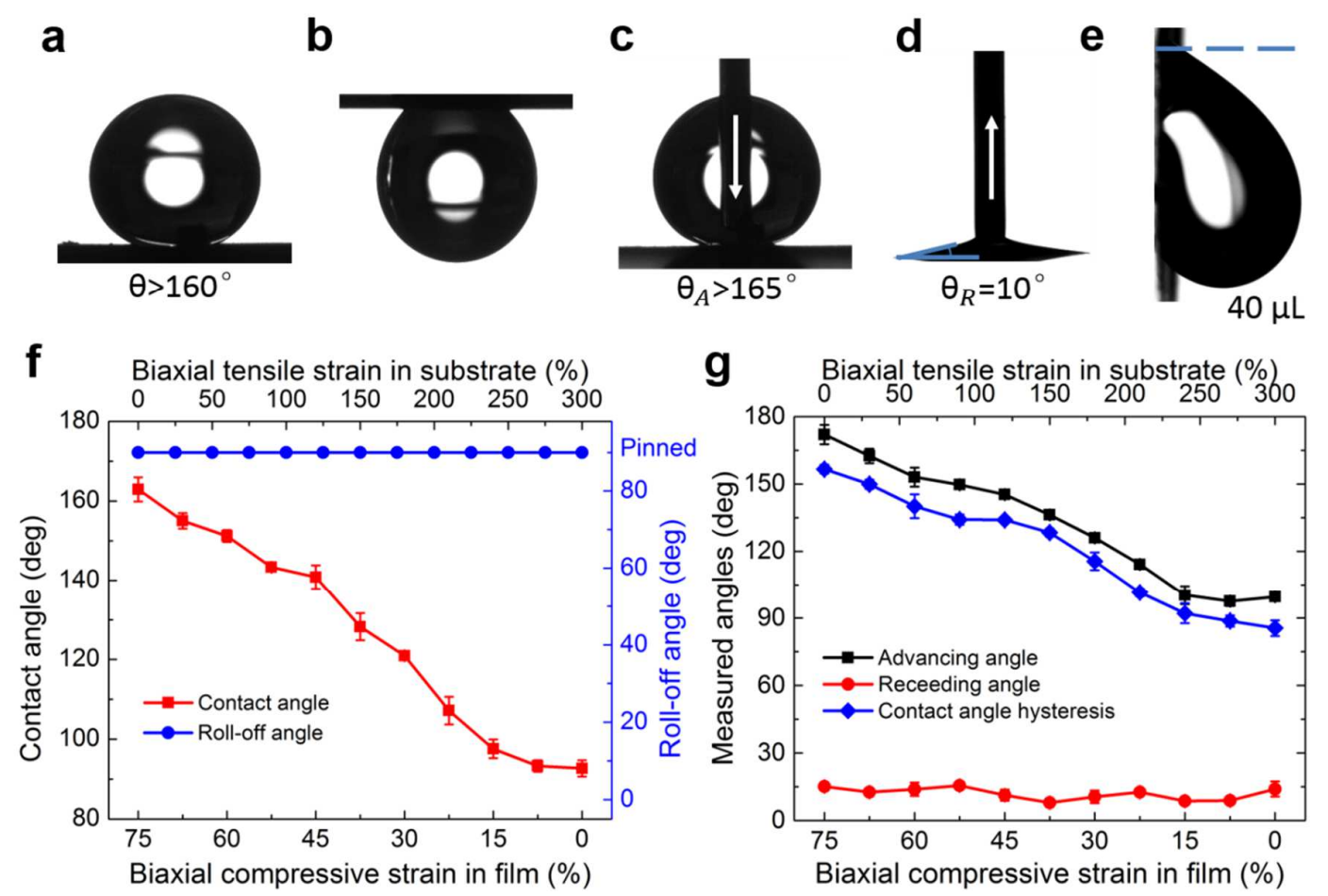

Fig. 3. Wetting properties of GP origami formed by sequentially relaxing biaxially prestrained substrate $\left(\varepsilon_{\text {pre } 1}=\varepsilon_{\text {pre } 2}=300 \%\right)$. (a) Shape of a water drop on the GP origami surface, indicating its large contact angle of $163^{\circ}$. (b) Shape of a suspending water drop $(5 \mu \mathrm{L})$ on the GP origami when the sample is turned upside down. (c) Advancing angle $\left(>165^{\circ}\right)$ of water droplet when adding water. (d) Receding angle $\left(=10^{\circ}\right)$ of water droplet when withdrawing water. (e) A large volume of water drop $(40 \mu \mathrm{L})$, with a gravity of $392 \mu \mathrm{N}$, suspended on the vertically placed surface. (f) The static contact angles of water drops on the GP as functions of the biaxial compressive strain in the GP or tensile strain in the elastomer substrate. (g) The advancing and receding angles and contact-angle hysteresis of water drop on GP as functions of the biaxial compressive strain in GP or tensile strain in the elastomer substrate. 
a

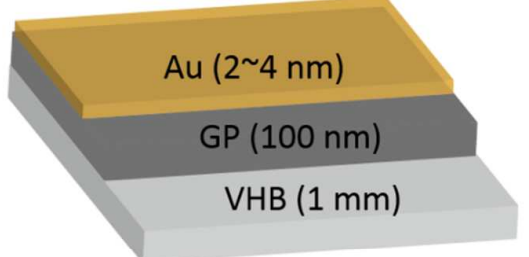

d

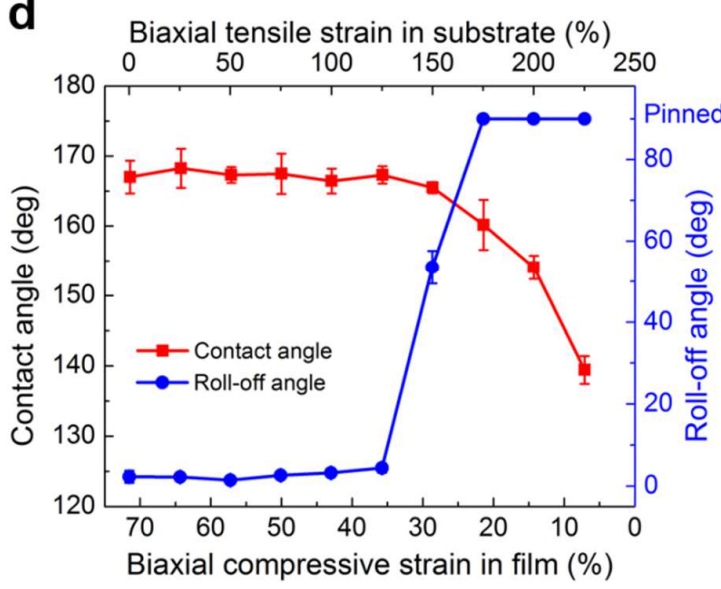

b

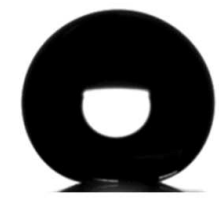

C

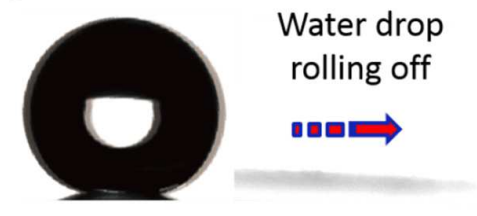

e

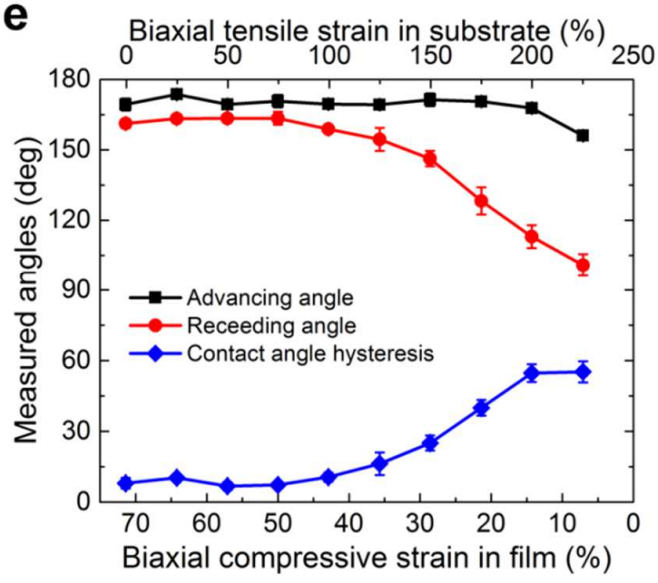

Fig. 4. Wetting properties of gold-coated GP origami formed by sequentially relaxing

biaxially pre-strained substrate $\left(\varepsilon_{\text {prel }}=\varepsilon_{\text {pre } 2}=250 \%\right.$ ). (a) Schematic illustration of the GP-Au films on elastomer substrate. The thickness of the gold film is $3 \pm 1 \mathrm{~nm}$. (b) Shape of a water drop on the GP-Au origami surface. (c) Rolling off of a water drop on the GP-Au origami surface at a tilting angle of $5^{\circ}$. (d) The static contact and roll-off angles of water drops on the GP-Au origami surface as functions of the biaxial compressive strain in the GP$\mathrm{Au}$ film or tensile strain in the substrate. (e) The advancing and receding angles and contactangle hysteresis of water drop on GP-Au origami as functions of the biaxial compressive strain in GP-Au film or tensile strain in the elastomer substrate. 

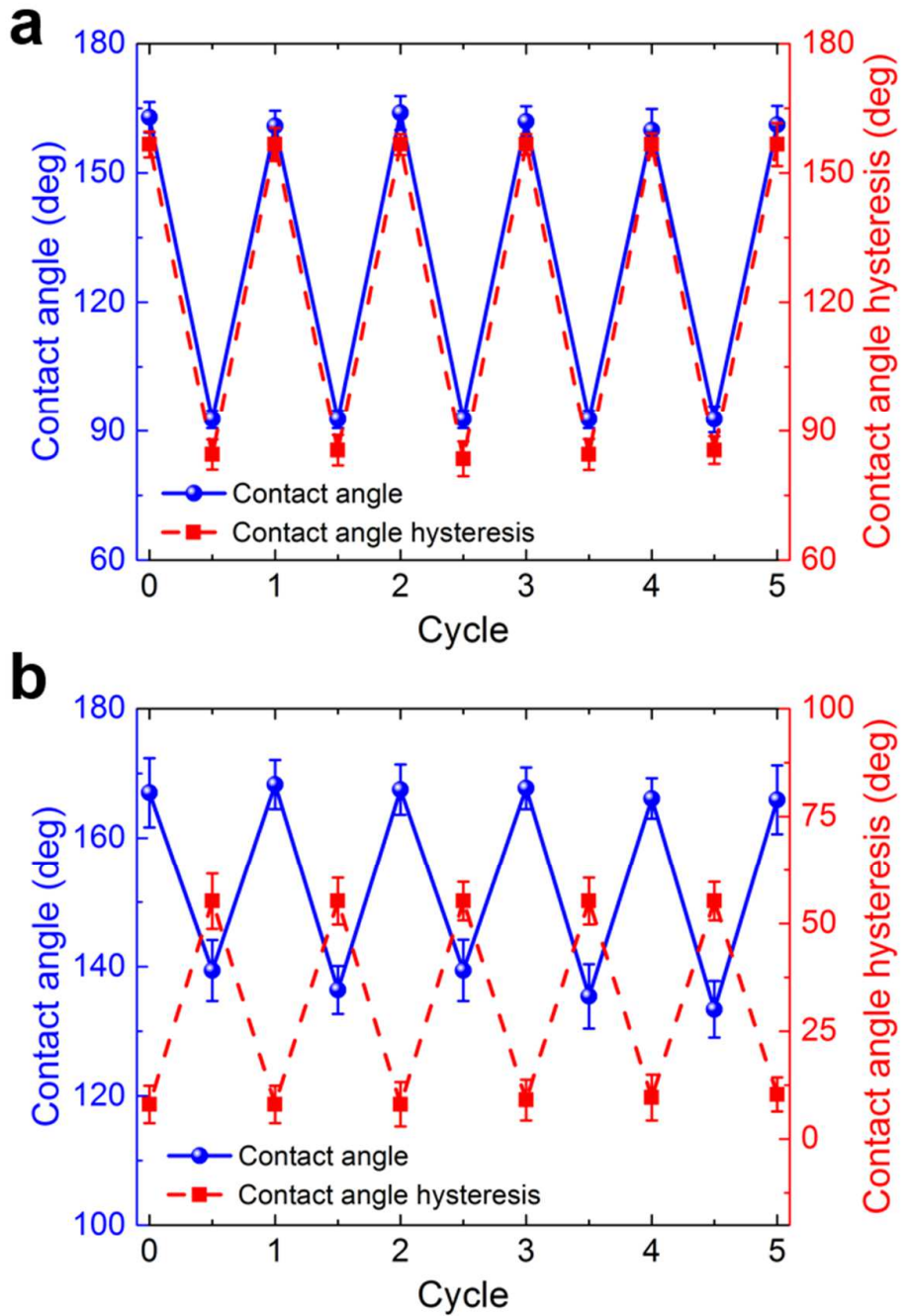

Fig. 5. (a) Reversibility and repeatability of tunable contact angle and contact angle hysteresis of GP origami ( $\varepsilon_{\text {prel }}=\varepsilon_{\text {pre } 2}=300 \%$ ). The elastomer substrate is gradually stretched from its original length to a relaxed state and then released to its original length. (b) Reversibility and repeatability of tunable contact angle and contact angle hysteresis of GP-Au origami $\left(\varepsilon_{\text {prel }}=\varepsilon_{\text {pre } 2}=250 \%\right.$ ). The variations of contact angle and contact angle hysteresis for both GP surface and GP-Au origami surfaces are reversible and can be repeated over successive cycles by stretching-releasing the elastomer substrate. 